\title{
Otium, liberté et création dans le roman d'artiste sandien
}

Otium, Liberty and Creation in George Sand's Artist Novel

\section{Gérard Peylet}

\section{OpenEdition}

\section{Journals}

Édition électronique

URL : https://journals.openedition.org/recherchestravaux/813

DOI : 10.4000/recherchestravaux.813

ISSN : 1969-6434

Éditeur

UGA Éditions/Université Grenoble Alpes

Édition imprimée

Date de publication : 1 juin 2016

Pagination : 85-105

ISBN : 978-2-84310-325-4

ISSN : 0151-1874

\section{Référence électronique}

Gérard Peylet, «Otium, liberté et création dans le roman d'artiste sandien », Recherches \& Travaux [En ligne], 88 | 2016, mis en ligne le 01 janvier 2017, consulté le 29 octobre 2021. URL : http://

journals.openedition.org/recherchestravaux/813; DOI : https://doi.org/10.4000/recherchestravaux 813 


\section{Otium, liberté et création dans le roman d'artiste sandien}

L'artiste a souvent besoin pour créer, de connaitre des temps de rupture, de recul. Il a besoin d'interrompre sa création pour mieux repartir. Il laisse à ce moment-là une place en lui que le philosophe Pierre Sauvanet nomme "l'insu' ${ }^{ }$. Arrêter d'écrire pour l'écrivain, de jouer pour le comédien sur un théâtre devant un public se révèle nécessaire avant de reprendre le travail, la création. Il y a quelque chose de positif qui sort de ce recul, qui a mûri de l'absence de contrôle dans cette rupture :

Il faut pouvoir arrêter de travailler, d'écrire pour l'écrivain, pendant un certain moment juste le temps d'en avoir envie à nouveau, de reprendre les choses où on les avait laissées, ou presque, parce que l'abandon momentané réalise malgré tout quelque chose. Il y a quelque chose qui sort du rien, qui mûrit en moi, comme une pâte qui lève quand elle repose. Question de rythme, là encore. Repos pour qu'il y ait élan - et inversement ${ }^{2}$.

Chez George Sand l'arrêt, le suspens n'est jamais vraiment synonyme d'absence de mouvement. Le suspens peut intervenir aussi bien à l'occasion d'un voyage que dans l'immobilité spatiale, dans un refuge. Mais la conscience des artistes représentés ou des personnages qui s'apparentent à des artistes n'est jamais interrompue. Elle profite de cette pause pour chercher de nouvelles orientations, s'ouvrir à de nouvelles expériences.

Le suspens ne signifie donc pas, bien au contraire, chez George Sand un vide de la conscience. C'est l'occasion pour ces artistes de vivre quelque chose

I. Au sujet de cette notion, «l'insu», lire le beau livre de P. Sauvanet, Linsu, une pensée en suspens, Paris, Arlea, 201 I.

2. Ibid., p. 156. 
d'unique : savoir s'oublier, oublier ses habitudes pour réussir ensuite quelque chose de neuf, savoir écouter les choses, les êtres. Avoir une oreille sur le monde, sortir de son narcissisme, savoir attendre qu'une inspiration vienne en son temps, à son rythme.

De nombreuses images expriment fréquemment dans l'œuvre ces deux dimensions de l'imaginaire sandien dans ces situations de rupture, de pause ou de suspens : l'ouverture et la médiation. L'ouverture, nous la retrouvons dans la représentation de l'espace, dans la psychologie des personnages en quête d'une transformation qui passe par un élargissement de leur vie, une connaissance de soi et des autres, donc une transformation de leur Weltanschauung, nous la retrouvons enfin dans de multiples thèmes comme le voyage ou la musique. Ces deux pôles sont indispensables à l'être sandien qui ne peut s'épanouir que dans une situation d'échange. Le suspens se révèle précisément indispensable pour l'artiste s'il veut connaître cette expérience de l'échange.

Le repli sur soi dans un lieu clos peut se révéler alors aussi nécessaire que le mouvement de déploiement hors du refuge et réciproquement. Les personnages du Château des Désertes choisissent le repli avant de repartir plus riches vers la société, les personnages de Teverino choisissent le voyage avant de revenir «transformés» chez eux. L'ouverture elle-même signifie souvent chez cet écrivain la transgression des barrières sociales, géographiques, spirituelles. Le repli lui-même peut favoriser une ouverture sur l'être, sur l'intériorité, sur la rêverie.

Nous allons examiner autour de cette question trois exemples, trois œuvres: Teverino, Le Château des Désertes, Consuelo. Il y sera question d'art plus que de littérature.

\section{Activité en suspens : Otium, nature et création dans Teverino ${ }^{3}$ (1846)}

L'otium n'est pas synonyme dans ce roman de désœuvrement puisque le repos, la promenade est au contraire une occasion d'enrichir ses sentiments à partir d'une remise en question, d'enrichir le goût esthétique de l'artiste (un dessinateur) au contact de la nature, de la liberté, d'une autre forme d'art.

Roman d'artistes, Teverino est aussi un roman de la frontière. Deux personnages un peu blasés, Sabina, une aristocrate, et Léonce, aristocrate et artiste (dessinateur), vont le temps d'une longue promenade de vingt-quatre heures franchir la frontière entre la France et l'Italie, mais aussi transgresser les limites et barrières sociales et culturelles, passer de l'oisiveté stérile, du désœuvrement, à une expérience des plus riches de l'otium: la révélation de l'autre et de soi, une

3. Teverino, roman publié dans La Presse en août-septembre i846. (Désormais T.) 
conception aussi renouvelée, vivifiée, de l'art. Deux personnages médiateurs, dont l'identité est assez floue et qui sont véritablement des êtres de la frontière et du passage, une jeune charmeuse d'oiseaux et un jeune chanteur vagabond d'origine italienne, propre à tous les talents, excellent musicien et chanteur, vont les accompagner dans cette expérience pleine de surprises et de révélations.

Léonce, l'artiste aristocrate, essaie d'abord de rassurer Sabina en ce qui concerne le caractère transgressif de cette promenade par rapport au code social des bonnes manières et il s'empresse en même temps de souligner le caractère poétique de cette expérience nocturne : «La poésie meurt au souvenir des convenances mondaines ${ }^{4}$ ( $T$, p. 635). Pour apprécier pleinement l'aventure, cette liberté qui ouvre l'esprit et le cœur des voyageurs, il faut oublier en effet le code social des "convenances mondaines".

La réflexion sur l'art dans cette aventure intervient spontanément chez Léonce, et Teverino, et Sabina parfois y prend part. Teverino, on l'a dit, incarne la figure du passeur. Lui-même réunit des qualités contradictoires et il passe de l'une à l'autre dans un mouvement dialectique. C'est un personnage du peuple qui possède en même temps des goûts aristocratiques. Son origine est mystérieuse et sa double ascendance populaire et aristocratique est d'ailleurs suggérée. De surcroit, ce vagabond est un artiste et l'on sait que l'artiste pour George Sand réconcilie souvent ces deux versants de la société que sont le peuple et l'aristocratie. Lorsque Léonce le rencontre dans la montagne, au bord d'un lac, Teverino se définit lui-même comme un être du changement :

Cette profession fut bientôt trouvée, c'est-à-dire qu'il s'en offrit cent, et que je n'en pris aucune exclusivement. J'avais l'amour du changement, la passion de la liberté, une curiosité effrénée pour tout ce qui me semblait noble et beau ( $T$, p. 614).

Ce personnage mobile, plus que double, aime le déguisement. Il se sert de celui-ci pour évaluer la perspicacité et la sensibilité de ceux qu'il rencontre. Dans la petite ville d'Italie où les voyageurs s'arrêtent la nuit, il se livre à une double expérience. Il va donner un concert tour à tour sous les traits d'un saltimbanque, misérable et grotesque, et sous ceux d'un chanteur aristocratique :

- Voyez ce que c'est que le préjugé, disait le bohémien. Cette foule imbécile ne se doute pas qu'elle a sifflé et applaudi le même homme; [...]

- Mes manières et mon langage étaient affectés, j'en conviens; mais enfin c'était toujours ma figure, ma voix, mon esprit, mon cœur, ma personne, mon être, en un mot, qui se cachaient sous ces apparences. (T, p. 648-649)

4. Toutes les citations que nous faisons de ce roman renvoient à l'édition de M.-M. Fragonard: George Sand, Vies d'artistes, Paris, Presses de la Cité, 1992. 
Teverino, être du passage, offre aux voyageurs qu'il guide dans cette expérience $\mathrm{du}$ franchissement de toutes les frontières - spatiale, sociale, psychologique une leçon. L'habitude, la routine, les conventions, nous conduisent à privilégier l'apparence sur l'être. Le vrai Teverino n'est pas plus dans l'image fugace du saltimbanque misérable que dans celle de l'aristocrate. Le vrai Teverino ne peut être saisi que dans la mobilité.

L'épreuve morale du passage de la frontière va conduire Sabina et Léonce à voir le monde, les êtres et les choses avec un regard libre, à ne pas juger les êtres sur leur apparence matérielle et sociale, à refuser le cloisonnement, l'enfermement. C'est, comme l'avait espéré Léonce, une véritable révolution que le personnage de Sabina va devoir faire : «Une révolution complète, inouïe, peut s'opérer aujourd'hui dans votre vie morale et intellectuelle» (T, p. 593). En effet Sabina et Léonce ne sont pas au même niveau lorsque l'aventure commence. Si Léonce n'est pas une figure de passeur, il est cependant l'initiateur de cette promenade qui va se transformer grâce à Teverino en expérience initiatique. Léonce est lui-même artiste. C'est lui qui choisit Teverino comme guide.

Le passage de la frontière, le temps d'une nuit, leur révélera que l'homme du peuple peut valoir et surpasser l'homme du monde dans le domaine des sentiments et de l'art. Passer la frontière, c'est accepter de voir les autres avec un regard neuf, c'est aussi accepter de changer soi-même.

Pour dire tous ces déplacements et toutes ces métamorphoses, George Sand met en œuvre dans cette "pure fantaisie» une poétique de la mobilité et de l'émotion. Toute l'action de ce petit roman repose sur le thème du voyage. L'otium source de création passe ici par le voyage, la mobilité, ce qui n'interdit pas des pauses pour réfléchir et méditer.

Teverino sert de fil conducteur à l'histoire et à la transformation des deux héros aristocrates. Il incarne bien évidemment le mouvement et la liberté. Pour lui la vie est dans le mouvement, et le bonheur, comme l'art, dans l'invention perpétuelle, l'acceptation du changement. Se fixer c'est se figer, c'est prendre le risque de passer à côté de la beauté des choses et des êtres, c'est prendre le risque de ne plus goûter la vie. Cet éloge de la liberté est aussi un éloge de la disponibilité. Teverino est disponible à toutes les émotions de la vie, celles qu'inspirent un spectacle de la nature, une œuvre d'art, une rencontre humaine.

Et justement la poétique de la mobilité repose dans ce roman sur deux motifs habilement entrelacés : celui de l'oiseau et celui du chant. L'oiseau et la musique traduisent mieux que tous les autres éléments à quel point cet espace

5. Sur cette question de la mobilité, on pourra lire avec intérêt l'ouvrage de C.-T. Doan Poisson sur l'œuvre autobiographique de George Sand, Histoire de ma vie : Poétique de la mobilité. Les lieux dans «Histoire de ma vie» de George Sand, Amsterdam-Atlanta, Rodopi, 2000. 
de la disponibilité que se révèle être la frontière implique une mobilité totale, absolue. L'espace de la frontière est ici propice à l'essor de l'imaginaire et de la sensibilité. Il incarne paradoxalement non pas la fermeture mais la mobilité absolue, la liberté suprême. Être mobile et disponible, c'est aussi accepter le danger.

Le deuxième motif que cette poétique de la mobilité exploite, c'est la musique, ou plus exactement le chant, à travers la voix de Teverino. La première fois que Teverino chante, c'est en présence de Léonce. La voix du jeune bohémien va libérer l'imagination de l'artiste aristocrate. Teverino souligne lui-même la spontanéité et la liberté infinie de la musique :

Le chant est de quelque dieu égaré sur les cimes de l'Apennin, qui l'aura confié aux échos, lesquels l'auront murmuré à l'oreille des pâtres et des pêcheurs; mais les paroles sont de moi [...] je suis improvisateur quand il me plaît de l'être [...]. Mes chants s'envolent de moi comme la flamme du foyer, je puis les renouveler et non les retenir. (T, p. 6II)

Le chant ignore les frontières. Symbole de l'un et du multiple, il se disperse, fluide et universel dans l'espace, il détient un pouvoir d'harmonie dans un mouvement que rien ne fige. La musique apparaît ici dans toute sa dimension de dépassement. C'est elle qui libère les sensibilités prisonnières des conventions, c'est elle qui ouvre l'être sur la profondeur. Elle incarne la mobilité suprême. Il n'est pas étonnant de la retrouver comme le motif de l'oiseau au cour de la structure imaginaire de ce roman. C'est un thème qui vient doubler la figure médiatrice du passeur. Elle apparaît comme une médiation idéale non seulement entre les consciences, mais entre la sensibilité masculine et la sensibilité féminine, entre les pays. Grâce à elle, la distinction entre le monde externe et le monde interne disparaît. Elle réunit le physique et le métaphysique, les sensations, les sentiments et l'esprit.

La frontière est un espace créateur de symboles et de mythes dans Teverino. Lieu de passage, ce mythe pose la question de l'altérité et de l'identité. Si elle peut être considérée comme un seuil que l'on franchit et à partir duquel notre vision du monde sera changée, la frontière sandienne n'est pas une clôture, elle est même le contraire d'une limite. Sa fonction n'est pas de retenir mais de faire passer, de provoquer la rencontre, l'échange qui va remettre en question bien des valeurs, sociales surtout. Toute une symbolique de la médiation et de l'ouverture se développe autour de ce thème.

Il faut noter qu'avant la promenade, Léonce, l'artiste aristocrate, manifestait des dispositions pour vivre pleinement la richesse de cette expérience. Il se définit ainsi devant Sabina : "Je suis artiste; je sens partout la présence de Dieu» (T, p. 58I). Avant de rencontrer Teverino, il perçoit à quel point la 
jeune montagnarde, Madeleine, est un être poétique. Il refuse les explications du curé qui craint que la jeune fille soit en proie au démon :

Pour apprivoiser à ce point des êtres libres et naturellement farouches, il faut une intelligence particulière, une sorte de secret magnétisme tout exceptionnel [...]. Ces rapports intimes sont des merveilles à nos yeux, parce que nous ne pouvons en saisir la loi naturelle, et le monde des faits physiques est plein de ces miracles qui nous échappent. ( $T$, p. 590-59I)

L'intelligence particulière que réclame Léonce est une forme d'intuition. Léonce trouve une confirmation de ce qu'il disait à Sabina et au curé en rencontrant Teverino au cœur de la montagne dans un cadre naturel de rochers sombres et de feuillages éclatants. Sa première réaction d'artiste, frappé de la perfection d'un semblable modèle, est de faire un croquis «de cet être bizarre qui offrait le plus beau type qu' un artiste ait jamais eu le bonheur de contempler" ( $T$, p. 609). Sa seconde réaction, conforme à ce que nous avons dit de l'otium dans l'introduction, est de fermer son album et de profiter du moment : "C'est bien assez pour moi [...] de le voir, de le sentir et de le graver dans ma mémoire» $(T$, p. 609). La suite marque le début de cette parenthèse dans la vie des personnages qui va allier la liberté, l'imagination et la création la plus spontanée. Teverino offre un chant italien à Léonce :

La voix du chanteur était magnifique, et aucun artiste en renom n'eût pu surpasser la franchise de son accent, la naïveté de sa manière, la puissance de son sentiment exalté. Léonce se crut transporté dans le golfe de Salerne ou de Tarente, sous le ciel de l'inspiration et de la poésie. ( $T$, p. 6IO-6II)

Teverino qui a avoué que les paroles étaient de lui, s'explique ensuite sur la spontanéité de sa création : "Je suis improvisateur quand il me plaît de l'être. Notre langue mélodique est à la portée de tous; et quand nous avons une idée, nous autres poètes naturels, enfants du soleil, l'expression ne se fait pas désirer longtemps» ( $T$, p. 6II).

Il faudra attendre le milieu de cette surprenante promenade, le franchissement de la frontière, pour que Teverino, dans un échange plus approfondi avec Léonce qui esquisse le portrait de Madeleine (en suscitant l'admiration de Sabina) se charge d'exprimer des idées chères à Sand sur la création :

Le véritable artiste est celui qui a le sentiment de la vie, qui jouit de toutes choses, qui obéit à l'inspiration sans la raisonner, et qui aime tout ce qui est beau sans faire de catégories. Que lui importe le nom, la parure et les habitudes de la beauté qui le frappe? [...] Vous êtes assez grand artiste, Léonce, pour faire de cette montagnarde blonde une sainte Élisabeth de Hongrie. (T, p. 639) 


\section{Activité en suspens : fuite, retraite et création dans Consuelo (I842-I843)}

\section{La fuite, le voyage, le chemin}

Comme dans Teverino, on retrouve le schème du voyage dans Consuelo. Les rapports entre le clos et l'ouvert qui marquent l'itinéraire initiatique de l'héroïne débouchent d'ailleurs dans l'Épilogue sur cette image finale du voyage infini. Dans Consuelo, le chemin a essentiellement deux valeurs. Quand l'héroïne s'enfuit du château des Géants pour gagner Vienne à pied (c'est l'épisode que nous allons étudier), il correspond à une période purificatrice de la vie de l'héroïne qui doit d'abord se libérer elle-même avant de libérer les autres. Sa rencontre avec le jeune Joseph Haydn est l'occasion pour Sand de méditer une fois encore, au sein de la fiction, sur le rapport entre la liberté et la création au sein d'une activité professionnelle suspendue.

Le premier point que la romancière met en valeur dans cette expérience, c'est le lien entre la liberté, la création, l'imagination et l'intuition, une intuition qui touche presque à la voyance. Il s'agit de la première pause dans ce voyage à deux. La scène nocturne qui représente un arrêt dans le voyage, correspond à un véritable moment d'abandon qui suit un autre mouvement - l'expansion, l'exaltation, l'élévation :

En songeant à Albert, elle s'était représenté cette faculté quasi surnaturelle qu'il avait souvent de voir et d'entendre à distance. Elle s'imagina fortement qu'à cette heure même il pensait à elle, et la voyait peut-être et, croyant trouver un allègement à sa peine en lui parlant par un chant sympathique à travers la nuit et l'espace, elle monta sur les pierres qui assujettissaient le pied de la croix. Alors, se tournant du côté de l'horizon derrière lequel devait être Riesenburg, elle donna sa voix dans toute son étendue pour chanter le verset du cantique espagnol : O Consuelo de mi alma, etc. [...] Consuelo redescendit de la pierre, où comme une madone elle avait dessiné sa silhouette élégante dans le bleu transparent de la nuit ${ }^{6}$. ( $C$, t. 2, p. 35$)$

La question que nous étudions réunit ensuite dans cet épisode le mouvement, la mobilité, la liberté, la création, la musique, mais au-delà de la musique, une réflexion plus large sur l'art, l'éducation artistique, l'apprentissage des langues. Le hasard joue un rôle de premier plan. Les deux artistes savent accueillir l'imprévu pour ouvrir leur talent et leur art sur de nouvelles expériences. Lorsque Joseph demande à Consuelo de lui apprendre l'italien, voici ce qu' elle répond :

6. Toutes les citations que nous faisons de ce roman renvoient à G. Sand, Consuelo, éd. S. Vierne et R. Bourgeois, t. 2, Grenoble, Aurore, I99I. (Désormais $C$, t. 2.) 
- Oh! de tout mon cœur, répondit Consuelo. J'aime qu'on ne perde pas un seul des précieux instants de la vie pour s'instruire; et comme on s'instruit soi-même en enseignant, il ne peut être que très bon pour nous deux de nous exercer à bien prononcer la langue musicale par excellence. Vous me croyez italienne, et je ne le suis pas, quoique j'aie très peu d'accent dans cette langue. Mais je ne la prononce vraiment bien qu'en chantant. ( $C$, t. 2, p. 33)

- Ce sera donc à la fois une leçon d'italien et une leçon de chant! s'écria Joseph. - Et une leçon qui durera cinquante lieues! pensa-t-il dans son ravissement.

Cependant le jeune musicien, qui souhaitait avec ardeur d'entendre la voix de la cantatrice, et qui n'en voyait pas venir l'occasion assez vite, la fit naître par une petite ruse. Il feignit d'être embarrassé de donner à l'à italien la franchise et la netteté convenables, et il chanta une phrase de Leo où le mot felicità se trouvait répété plusieurs fois. Aussitôt Consuelo, sans s'arrêter, et sans être plus essoufflée que si elle eût été assise à son piano, lui chanta la phrase à plusieurs reprises. À cet accent si généreux et si pénétrant qu’aucun autre ne pouvait à cette époque, lui être comparé dans le monde, Joseph sentit un frisson passer dans tout son corps, et froissa ses mains l'une contre l'autre avec un mouvement convulsif et une exclamation passionnée. ( $C$, t. 2, p. 33)

La rupture, l'activité en suspens qui caractérise selon nous, chez cet auteur romantique, l'otium favorise des moments exceptionnels de recueillement, de méditation et de liberté ou d'abandon artistique. La romancière souligne ellemême que c'est durant cette période de sa vie que le jeune compositeur a acquis «les meilleures notions de musique qu'il eût encore eues dans sa vie» $(C, \mathrm{t}$. 2 , p. 49) : «Durant les longues haltes qu'ils firent dans les beaux jours, sous les solitaires ombrages du Boehmer-Wald, nos jeunes artistes se révélèrent l'un à l'autre tout ce qu'ils possédaient d'intelligence et de génie» ( $C$, t. 2, p. 49).

Le voyage, mais aussi la halte dans le voyage - cette pause dans l'espace du voyage et dans le temps, presque une suspension - favorise la révélation artistique, l'échange, l'ouverture et chez Consuelo le désir d'aider le génie de Haydn à s'éveiller :

Voyons donc un peu vos compositions! Malgré mes longues et sévères études de contre-point avec un aussi grand maitre que le Porpora, ce que j'ai appris ne me sert qu'à bien comprendre les créations du génie, et je n'aurai plus le temps, quand même j'en aurais l'audace, de créer moi-même des œuvres de longue haleine; au lieu que si vous avez le génie créateur, vous devez suivre cette route, et ne considérer le chant et l'étude des instruments que comme vos moyens matériels. ( $C$, t. 2, p. 49)

\section{Consuelo est aussi peu désireuse que Joseph de voir finir leur voyage :}

Elle était trop artiste par toutes les fibres de son organisation, pour ne pas aimer la liberté, les hasards, les actes de courage et d'adresse, le spectacle continuel et varié de cette nature que le piéton seul possède entièrement, enfin toute l'activité romanesque de la vie errante et isolée. ( $C$, t. 2, p. 96) 
Cette phrase est capitale. Elle souligne à quel point cette position à l'écart de l'activité sociale, cette solitude que l'on savoure, est profitable pour retremper ses forces, méditer. Cette situation d'écart est l'occasion pour l'âme de l'artiste de "goûter une suspension d'activité qui est comme le profond sommeil d'un corps vigoureux" ( $C$, t. 2, p. 97). Cet écart favorise aussi l'improvisation. Lorsque Consuelo doit remplacer l'organiste malade dans un petit village, à l'occasion d'une fête, elle doit trouver de nouveaux airs, des solos :

Consuelo chercha et trouva dans sa mémoire un chant religieux du Porpora qui s'adaptait au ton et aux paroles du solo exigé. Elle l'écrivit sur son genou, et le répéta à la hâte avec Haydn, qui se mit ainsi en mesure de l'accompagner. ( $C$, t. 2, p. Io9)

Ce temps de loisir permet aussi à l'artiste de profiter de tout ce que le hasard met à sa portée. Ils sont accueillis un soir par le chanoine, bon musicien qui leur fait entendre des fragments de plusieurs vieux maîtres allemands.

Cette audition ne fut pas sans intérêt pour Consuelo; et bientôt, ayant trouvé sur le clavecin un gros livre de cette ancienne musique, elle se mit à le feuilleter et à oublier la fatigue et l'heure qui s'avançait, pour demander au chanoine, de lui jouer, avec sa bonne manière nette et large, plusieurs morceaux qui avaient frappé son esprit et ses yeux. ( $C$, t. 2, p. I27)

Rien n'est jamais programmé dans cette forme de création en liberté qui touche à la fois, l'esprit, les sens et le cœur. C'est le sens que Consuelo donne au mot d'ivresse, d'exaltation pour qualifier cette forme de création libre :

Vous m'avez fait tant chanter (dit-elle au chanoine), et vous m’avez tant poussé à l'exaltation en manifestant la vôtre, que je suis peut-être un peu hors de moi. Si j'éprouve une sorte d'ivresse, c'est votre faute; mais soyez sûr que la vérité parle dans ces ivresses-là, parce qu'elles sont nobles et développent en nous ce que nous avons de meilleur. Elles nous mettent le cœur sur les lèvres, et c'est mon cœur qui vous parle en ce moment. ( $C$, t. 2, p. I42)

Cette phrase capitale encore a valeur de manifeste pour George Sand.

\section{Liberté et création en prison}

Il nous reste à dire un mot d'une autre expérience que va connaître l'artiste Consuelo, celle de la prison. Consuelo va parfaire encore dans ce lieu son éducation d'artiste en abordant la composition. C'est à travers l'épreuve initiatique de la prison de Spandow qu'elle va devenir une plus grande artiste en composant des airs populaires ou religieux - ce qui n'est pas contradictoire pour Sand - et en libérant dans une nuit déjà symbolique une part profonde du génie musical qui est en elle : 
Ses soirées si redoutables d'abord étaient devenues ses heures les plus agréables; et les ténèbres, loin de lui causer l'effroi qu'elle en attendait, lui révélèrent des trésors de conception musicale, qu'elle portait en elle depuis longtemps sans avoir pu en faire usage et les formuler, dans l'agitation de sa profession de virtuose? ${ }^{7}$ ( $C$, t. 3, p. I46)

Cette pause n'a rien de décoratif et de gratuit. L'héroïne qui s'abandonne aux puissances de la nuit vit à son insu un progrès intérieur. La nuit permet à l'héroïne d'élargir son univers, de repousser ses limites, de percevoir de nouveaux liens entre les êtres, entre l'être et le cosmos. Consuelo, le roman opéra de George Sand, est d'ailleurs jalonné de ces moments cruciaux. C'est l'épreuve de la prison qui permet à l'héroïne de libérer pendant la nuit une part profonde du génie musical qui est en elle et qu'elle avait méconnu jusque-là.

La menace des ténèbres s'inverse en une nuit bienfaisante. L'inversion des valeurs diurnes qui étaient valeurs de l'étalement, de "l'agitation», mais aussi de la séparation et du morcellement, entraîne la valorisation des images de l'intériorité, de la profondeur. La prison, en tant que suspension d'activité, libère le génie et la créativité de l'artiste.

Je chante tous les soirs pendant deux heures, et il me semble que je fais des progrès. À quoi cela me servira-t-il? Les voûtes de mon cachot me répondent, elles ne m'entendent pas... Mais Dieu m'entend, et quand j'ai composé un cantique que je lui chante dans la ferveur de mon âme, j'éprouve un calme céleste, et je m'endors presque heureuse. Il me semble que du ciel on me répond, et qu'une voix mystérieuse me chante dans mon sommeil un autre cantique plus beau que le mien, que j'essaie le lendemain de me rappeler et de chanter à mon tour. ( $C$, t. 3, p. 173)

De Venise au château des Invisibles, en passant par Vienne, Berlin, le château des Géants et la prison de Spandow, Consuelo, la voyageuse, va approfondir sa vocation musicale, découvrir que la musique constitue un langage complet au-dessus de tous les autres, supérieur à la parole humaine toujours incomplète, qu'elle est communication d'êtres dans l'échange d'une émotion partagée, qu'elle va profondément d'une conscience à une autre, d'une âme à une autre, qu'elle est à la fois don et réception, don infini et indéfini dont le sens n'est jamais figé. Son voyage à pied dans le Tyrol en compagnie du jeune Joseph Haydn est en tout cas exemplaire du lien qui unit la liberté et la création ainsi que plus paradoxalement son séjour dans la prison de Spandow.

7. Toutes les citations que nous faisons maintenant renvoient à G. Sand, Consuelo, La comtesse de Rudolstadt, t. 3, Grenoble, Aurore, 199i. (Désormais $C$, t. 3.) 


\section{Otium et éducation artistique dans Le Château des Désertes (I85I)}

Peu de romanciers ont autant que George Sand représenté des vies d'artistes, et parmi celles-ci, les acteurs, les musiciens et les cantatrices, occupent une place de premier plan. Le Château des Désertes a été écrit après la grande désillusion politique de 1848, à un moment où Sand repliée à Nohant va se détourner de la voie la plus politique de son œuvre pour se consacrer à l'écriture des romans champêtres, sans abandonner la veine des romans d'artistes qui lui a toujours été chère. C'est à cette époque que la petite société de Nohant qui pratique un otium littéraire des plus actifs trouve son centre autour de l'expérience théâtrale : celle du théâtre de marionnettes de Maurice ${ }^{8}$ d'abord, celle des pièces que l'on joue avec les visiteurs et les amis. Nohant était devenu depuis I846-I847 une sorte de laboratoire expérimental de la technique d'improvisation.

George Sand aime les théories, mais n'en fait pas son métier et laisse à ses fictions et à ses personnages le soin de parler à sa place. Elle n'est pas doctrinaire, elle refuse tout système qui enferme. Il n'y a pas chez elle «de définition unique de l'art ni de stricte limitation de ce qu'est un artiste», prévient MarieMadeleine Fragonard dans sa présentation à l'édition Vies d'artistes dans la collection Omnibus qui réunit neuf romans?.

C'est ce que nous découvrons avec Le Château des Désertes qui est peutêtre le roman où l'auteur analyse de plus près le double lien qui unit l'acteur à l'art et à la vie. Nous voyons dans ce roman comment George Sand aborde la maîtrise de l'outil et de la technique en suivant le modèle de la commedia dell'arte, comment elle a réfléchi au problème de la double formation parallèle du talent et du moi, nous y découvrons enfin la quête du sens de la vie soutenue par un idéal d'unité et d'harmonie.

L'espace choisi pour cette expérience unique, extrêmement forte, est un lieu clos, un château. Ce lieu représente un espace idéal de libération et d'épanouissement. Le château d'ombre gothique s'ouvre ici aux lumières de l'intelligence et du cour. Cet espace particulier, de la marge, de la rupture, s'oppose dans ce roman à une société où l'homme se disperse dans les apparences et les illusions. C'est le lieu idéal de l'apprentissage par le jeu, le divertissement, où le jeune artiste se concentre pour mieux se connaître et développer ses facultés avant de revenir à la société, une fois qu'il sera assez solide pour ne plus s’y perdre

8. Maurice, le fils de George Sand.

9. M.-M. Fragonard, «Présentation », dans G. Sand, Vies d'artistes, Paris, Presses de la Cité, I992, p. III et IV. Toutes les citations que nous donnerons du Château des Désertes (désormais $C D)$ renverront à cette édition. 
et s’y gâter. Le château dans ce roman de I85I est placé sous le double signe de la métamorphose et de l'harmonie. L'expérience théâtrale s'y déroule loin des regards indiscrets. Qu'importe si les gens simples du pays, les profanes, imaginent des histoires sataniques, de "gros secrets", l'espace du château contient l'espace de l'éducation artistique.

La clôture ici est nécessaire à l'initiation des jeunes gens au monde de l'art. Le château d'ombre, satanique encore aux yeux des profanes, va se révéler un château de lumière et d'élévation spirituelle pour les initiés. Le mystère qui l'habite est positif, source d'impressions fécondes pour les artistes : «Le mystère dont nous réussissons à nous entourer, me dit Cécilia, donne un nouvel attrait à nos études et à nos fêtes nocturnes» ( $C D$, p. 930), car la fête fait partie de cette parenthèse, de cette activité sociale, officielle ou conventionnelle suspendue. Le château mystérieux suscite chez le narrateur artiste quand il rejoint ce lieu, par hasard, une émotion poétique, stimule sa sensibilité :

[...] je ne pouvais me défendre d'une émotion analogue à celle que nous fait éprouver la lecture d'un conte fantastique d'Hoffmann, en rapprochant de ces charmantes figures si candides, si enjouées, si heureuses en apparence, les récits bizarres et les diaboliques commentaires de ma vieille hôtesse [...] et je mourais d'envie d'agir comme un écolier, c'est-à-dire d'aller chercher quelque aventure poétique ou ridicule sous les murs du vieux château. ( $C D$, p. 904-905)

La métamorphose ne concerne pas seulement le château lui-même, mais également ses habitants. Ce lieu idéal pour l'expérience théâtrale et artistique va former, transformer, métamorphoser les jeunes artistes qui ont la chance de participer à cette expérience.

Le Château des Désertes est incontestablement un roman nourri de réflexion artistique comme le reconnait George Sand dans la «Notice»: «Le Château des Désertes est une analyse de quelques idées d'art plutôt qu'une analyse de sentiments» (CD, p. 85I). Ce roman propose d'abord une technique d'apprentissage qui semble prendre le contre-pied du Paradoxe sur le comédien de Diderot. On connaît la pensée polémique de Diderot ${ }^{\mathrm{TO}}$ sur le théâtre : celui-ci est présenté

IO. La réflexion de Diderot dans le Paradoxe sur le comédien est d'ailleurs très éloignée de la réflexion de cet auteur sur la place du sentiment dans la création artistique que l'on rencontre aussi bien dans La Piété filiale, les Pensées détachées sur la peinture ou le Salon de 1763. Dans ces derniers textes Diderot défend contre l'artifice les valeurs de sincérité et de véracité qui lui paraissent seules capables de régénérer l'art et de lui donner sa véritable grandeur. George Sand n'a certainement pas été insensible aux idées de cette critique sentimentale du XVIII ${ }^{\mathrm{e}}$ siècle qu'elle a trouvée aussi bien chez Diderot que chez Rousseau avant de les rencontrer ensuite chez Madame de Staël. Comme ses prédécesseurs elle va prôner l'élévation de l'inspiration, la sincérité esthétique, l'accord entre le caractère de l'artiste et son œuvre, la rencontre entre la sincérité de l'artiste et l'attendrissement du spectateur. 
comme un instrument qu'on programme en toute lucidité pour fabriquer des émotions. L'art est entièrement distinct de la nature, le vrai comédien se défie de son enthousiasme et est capable de se dédoubler, il doit rendre scrupuleusement les signes extérieurs des sentiments. Seule la lucidité permet d'atteindre la perfection et de recréer la nature.

George Sand n'oppose pas du tout l'art et la nature lorsqu'elle propose l'improvisation comme technique d'apprentissage. Elle choisit de tracer le portrait du personnage d'acteur ou de metteur en scène, au moment où il se montre le plus artiste, lors du jaillissement de son talent. La maîtrise de la technique d'interprétation passe par la nécessité de l'émotion, par la priorité accordée au sentir. Le jugement qui était la qualité essentielle demandée par Diderot n'est pas écarté, mais il intervient après le jaillissement de l'émotion et de l'enthousiasme.

Sous la direction du vieux Boccaferri, ancien acteur à la retraite, devenu marquis grâce à un héritage, riche seigneur rajeuni par l'exercice de l'art théâtral, les acteurs entrainés par lui comme Célio, Stella, Salvator le nouveau venu, découvrent que l'intuition quand elle atteint son but va beaucoup plus loin que le travail et le jugement réunis. Boccaferri qui «eut peur», lors de la dernière représentation de Don Juan, de la statue du commandeur qu' il n'avait pas vue à la répétition de la veille, avoue :

J'ai réellement eu peur. Je suis content, notre essai réussit, mes enfants; voilà que l'émotion nous gagne. Pour moi, c'est déjà fait, et quand vous en serez tous là, vous serez tous de grands artistes!... (CD, p. 915)

C'est le retournement du Paradoxe sur le comédien, mais il faut bien voir que «l'essai» dont parle Boccaferri constitue une méthodologie d'apprentissage entre théorie et pratique constamment mêlées. Seule l'improvisation a des chances de faire jaillir le génie qui sommeille chez un grand interprète, de faire jaillir le meilleur et le plus beau de lui-même. Évidemment Boccaferri réserve son enseignement à des êtres qui en sont déjà dignes, à des artistes. L'improvisation "gouvernée " par un metteur en scène de génie attentif à tous les signes de révélation du beau et du vrai favorise l'épanouissement créateur de l'artiste. Boccaferri explique à Salvator ce qu' est ce laboratoire expérimental qu'il dirige avec souplesse :

Vous avez déjà compris que nous inventions un thêâtre d'une nouvelle forme et complètement à notre usage. Nous prenons le premier scénario venu, et nous improvisons le dialogue, aidés des souvenirs du texte. Quand un sujet nous plait, comme celui-ci, nous l'étudions pendant quelques jours en le modifiant ad libitum. Sinon, nous passons à un autre, et souvent nous faisons nous-mêmes le sujet de nos drames et de nos comédies, en laissant à l'intelligence et à la fantaisie de chaque personnage le soin d'en tirer parti. Vous voyez déjà qu'il ne s'agit pour nous que 
d'une chose, c'est d'être créateurs et non interprètes serviles. Nous cherchons l'inspiration, et elle nous vient peu à peu. ( $C D, \mathrm{p}$. 918)

Il n'y a rien de systématique dans ce retournement du Paradoxe sur le comédien. Si l'improvisation est nécessaire, tout comme l'inspiration, Boccaferri reconnait "qu'il faut de l'ordre même dans l'inspiration». Une sorte d'équilibre s'établit chez lui entre la réflexion et l'intuition. Les deux qualités collaborent sans cesse : "Il se livrait à une consciencieuse étude de l'art, et il faisait d'admirables élèves en cherchant lui-même à mettre en pratique des théories qui avaient été le rêve de sa vie entière» $(C D$, p. 918). Le directeur de troupe conseille à ses acteurs de ne pas perdre le temps de l'entracte et de mettre à profit ce moment pour soumettre les émotions rencontrées au contrôle de l'intelligence : "Tandis qu'il repose ses membres et sa voix, il faut que son intelligence continue à travailler, qu'il résume ses émotions récentes» ( $C D, \mathrm{p}$. 924).

Précisons qu'il s'agit d'une école de théâtre non officielle, non institutionnelle, plus que de théâtre. Le public est absent. La troupe représente son propre public et tout repose sur la complicité et le jeu, tout est gratuit. Cette pratique théâtrale restreinte au temps de l'apprentissage doit permettre ensuite le retour des acteurs, surtout celui de Célio, vers un théâtre de convention : «Il est vrai que nous employons ici, pour notre propre satisfaction, des moyens naïfs dont le charme serait perdu sur un grand théâtre. Nous plantons de vrais arbres sur nos planchers et nous mettons de vrais rochers jusqu'au fond de notre scène " $(C D$, p. 914). Le but pour Boccaferri dans cet apprentissage est de susciter le jeu authentique à partir d'une situation d'authenticité : «que le théâtre soit vrai, tous les acteurs seront vrais [...]. Il y a peu d'acteurs vrais, et tous devraient l'être! Qu'est-ce qu'un acteur, sans cette première condition essentielle et vitale de son art?» ( $C D$, p. 919-920) C'est en retrouvant une situation d'authenticité que l'acteur est susceptible de redevenir, comme tout véritable artiste, "un médium révélateur de qualités qui le dépassent ${ }^{\mathrm{II}} »$.

L'intention de Sand est claire. Elle refuse tout ce qui s'établit sur le renoncement à l'authenticité. L'acteur qui joue est sincère dans son rôle. L'acteur interprète ne peut devenir à son tour créateur que s'il parvient au jeu authentique. Pour Diderot le comédien est un pantin merveilleux dont le poète tire les ficelles et auquel il indique à chaque ligne la véritable forme qu'il doit prendre. Pour Sand, l'acteur est un créateur qui s'impose au rôle, puisqu'il en est le créateur, qui transmet au rôle la vie qu'il a puisée en lui-même. C'est grâce à l'artiste interprète qu'un personnage littéraire comme Don Juan se révèle chaque fois autre et unique. L'acteur peut être un véritable créateur

II. M.-M. Fragonard, «Présentation», ouvr. cité, p. IV. 
non parce qu'il est un instrument docile, mais parce qu'il a d'abord la richesse intérieure de l'intelligence et des sentiments que l'exercice de son art révèle. C'est un créateur, car il a le don d'agir sur la sensibilité d'autrui en puisant dans les richesses que sa propre sensibilité a su emmagasiner.

Cette technique de l'improvisation connaittra, d'après Boccaferri, ses limites sur un vrai théâtre face à un vrai public :

Ici, nous sommes libres de la lettre, et l'esprit d'improvisation nous ouvre un champ illimité de créations délicieuses. Mais, lors même qu'en public vous serez esclave d'un texte, un geste, une expression de visage suffiront pour rendre votre intention. Ce sera plus difficile, mes enfants! car il faudra tomber juste du premier coup, et résumer une grande pensée dans un petit effet. ( $C D$, p. 925)

Le château des Désertes devient ainsi le lieu idéal du projet le plus ambitieux qui soit pour un artiste, celui d'une rénovation artistique. C'est ce qu'explique le vieux maître à ses disciples : "Mais toi, Célio, mais vous, Stella, Béatrice, Salvator, vous êtes jeunes, vous êtes unis, vous comprenez l'art maintenant, et vous pouvez, à vous quatre, tenter une rénovation» $(C D$, p. 920). Ce projet a fait comprendre aux jeunes artistes qu'il ne fallait pas confondre art et artifice, qu'une certaine émotion est irremplaçable chez le créateur et chez le récepteur, que le sens d'une œuvre - on appréciera la modernité de George Sand - n'est jamais fermé : «Ne te gêne pas, mon enfant, nous sommes ici pour interpréter plutôt que pour traduire [...]. Don Juan est un chef-d'œuvre [...] mais c'est un chef-d'œuvre en plusieurs versions» ( $C D$, p. 930).

La métamorphose ne se limite pas dans ce roman de l'art au lieu et au jeu des comédiens. Elle concerne aussi tout leur être. Cette période d'apprentissage est nécessaire à double titre. L'école de Boccaferri est une école de formation pour devenir artiste et pour devenir un homme. L'école d'art et l'école de vie sont inséparables. C'est d'ailleurs en découvrant les défauts de Célio, que le vieux Boccaferri aura l'idée de créer cet apprentissage exceptionnel au cœur du château des Désertes. Quand Célio se lance en effet dans la carrière de chanteur, il rencontre des difficultés liées à son talent mais surtout à sa personnalité trop narcissique :

C'est en voyant combien ce jeune homme avait peu de conscience que j'ai senti le prix de la conscience dans l'art lyrique, aussi clairement que je le sens dans l'art de la peinture et dans tous les arts [...] il a manqué à ce qu'il y a de plus sacré [...] il a manqué à l'amour et au respect de son art [...] j'ai compris que l'absence de charme et de puissance de ce jeune homme tenait à sa vanité, à son besoin d'être admiré, à son peu d'amour pour l'œuvre qu'il chantait, à son manque de respect pour l'esprit et les traditions de son rôle. Il s'est nourri toute sa vie, j'en suis sûr, de l'idée qu'il ne pouvait faillir et qu'il avait le don de s'imposer. Probablement c'est un enfant gâté. ( $C D$, p. 864) 
«[C]'est un enfant gâté». Cette remarque de Boccaferri constitue bien le point de départ de l'expérience que cette troupe sans public va vivre, à l'écart de la société, dans le vieux château. L'éducation morale y est inséparable de l'éducation artistique. George Sand est persuadée que «l'improvisation théâtrale» que le maître demande à ses élèves favorise l'épanouissement du talent créateur de l'artiste à travers une meilleure connaissance de soi. Devenir un bon interprète est une façon de se découvrir aux autres en se découvrant soi-même. Une fois de plus on se rend compte que George Sand n'aime pas séparer la question du Beau de la question du Bien. Elle a besoin de lier le domaine de l'esthétique aux valeurs spirituelles. L'esthétique de Sand est aussi une éthique. Puisqu'il rend «vrai»l'idéal, l'artiste est par son art le propagateur de la vérité. L'énergie de la création est son principe de vie et se renouvelle de création en création. L'énergie est ce par quoi l'artiste peut s'engager tout entier dans son art. Cette énergie ne peut être aux yeux de Sand qu'une vertu qui implique le renoncement à l'égoïsme, au mensonge.

Boccaferri dévoile devant Salvator les raisons morales qui l'ont poussé à prendre en charge les enfants de la Floriani, personnage de grande actrice, imaginé par Sand quelques années avant Le Château des Désertes ${ }^{12}$ :

J'ai dû tant à leur mère! mais ce n'est pas avec de l'argent seulement que je puis m'acquitter envers celle qui ne m'a pas secouru seulement avec de l'argent : elle m'a aidé et soutenu avec son cœur, et mon cœur appartient à ce qui survit d'elle, à ces nobles et beaux enfants qui sont désormais les miens [...]. Puisque la Providence m'en fournit les moyens, je veux qu'ils aient les coudées franches dans la vie, et je les ai tout de suite appelés à moi pour qu'ils ne me quittent que le jour où ils seront assez forts pour se lancer sur la grande scène de la vie comme artistes; car c'est la plus haute des destinées, et, quelle que soit la partie que chacun d'eux choisira, ils auront étudié la synthèse de l'art dans ses détails auprès de moi [...]. Passez-moi cette vanité $[\ldots]$. Je crois qu’à force de réflexions et d'expériences je suis arrivé à tenir dans mes mains la source du beau et du vrai. (CD, p. 93I)

Entre ces entités abstraites, beau, vrai, bien, juste, s'opère souvent chez George Sand une série d'équations qui révèle une volonté de ne pas couper le développement artistique du développement moral. L'enseignement que dispense Boccaferri ne peut d'ailleurs vraiment porter ses fruits que s'il est dispensé à des natures riches et généreuses : «J'ai la certitude qu'on ne fait rien avec rien, et que l'enseignement n'est utile qu'aux êtres richement doués par la nature»

I2. Le Château des Désertes constitue d'ailleurs la suite du roman Lucrezia Floriani écrit en I846, roman d'analyse, roman de la passion malheureuse, dans lequel l'héroïne, Lucrezia Floriani, s'est retirée de la scène, après une brillante carrière, pour se consacrer à l'éducation de ses quatre enfants. Ce sont ces enfants que l'on retrouve dans Le Château des Désertes, aux côtés de Boccaferri, ancien protégé de Lucrezia, et de sa fille. 
( $C D$, p. 937). Célio, l'un des enfants les plus brillants de la Floriani et l'un des plus brillants élèves de Boccaferri, a compris cette double dimension de l'apprentissage qu'il reçoit :

Oui, c'est la vérité, dit Célio, tu m'as appris à me connaitre. Tu m'as rendu l'orgueil en me guérissant de la vanité. Il me semble que, chaque jour, ta fille et toi vous faites de moi un autre homme. Je me croyais envieux, brutal, vindicatif, impitoyable. J'allais devenir méchant parce que j'espérais à l'être; mais vous m’avez guéri de cette dangereuse folie, vous m’avez fait mettre la main sur mon propre cœur. Je ne l'eusse pas fait en vue de la morale, je l'ai fait en vue de l'art, et j'ai découvert que c'est de là (et en parlant ainsi Célio frappa sa poitrine) que doit sortir le talent. (CD, p. 937-938)

École d'art et école de vie, l'apprentissage du théâtre a transformé l'élève comme il transforme le maître : «j'étais vivement ému; j’écoutais Célio avec attendrissement; je regardais le marquis de Balma avec admiration. C'était un autre homme que celui que j'avais connu; ses traits mêmes étaient changés » (CD, p. 938). George Sand insiste sur cette naissance d'un homme nouveau qui passe par une transformation physique. L'artiste tendu vers l'idéal du beau et du vrai finit par faire jaillir le beau de lui-même. On peut alors parler d'une trans-figuration au sens littéral du mot.

Le but principal de l'apprentissage n'est d'ailleurs pas limité au seul exercice futur du théâtre. Stella, la sœur de Célio, dit à son tour à Salvator :

Vous n'avez pas compris notre cher maitre Boccaferri, si vous croyez qu'il n'a en vue que de nous faire chanter. Non, ce n'est pas là sa pensée. Il pense que ces essais dramatiques, dans la forme libre que nous leur donnons, sont un exercice salutaire au développement synthétique (je me sers de son mot) de mes facultés d'artiste, et je crois bien qu'il a raison, car depuis que nous faisons cette amusante étude je me sens plus peintre et plus poète que je ne croyais l'être. ( $C D$, p. 95I)

Apprentissage du théâtre, apprentissage de la vie - le but principal de l'éducation que Boccaferri donne aux enfants de la Floriani, est l'épanouissement de l'être, l'agrandissement de l'âme :

[...] je me sentais grandir au contact de ces fraîches imaginations d'artistes dont le vieux Boccaferri était la clé, le lien et l'âme. Je dois dire que Lucrezia Floriani avait bien connu et bien jugé cet homme, le plus improductif et le plus impuissant des membres de la société officielle, le plus complet, le plus inspiré, le plus artiste enfin des artistes. ( $C D$, p. 953)

Salvator le peintre, qui est également le héros narrateur de cette histoire, sent lui aussi son art se régénérer au contact de cet étonnant professeur : «En barbouillant de grossiers décors (car il peignait fort mal), il épanchait dans mon sein un flot d'idées lumineuses qui fécondaient mon intelligence et dont je sentirai toute ma vie la puissance génératrice» ( $C D$, p. 953). C'est 
par le sentiment, par la fibre sensible, que l'artiste sandien reçoit la lumière intellectuelle et morale. On voit que le sentiment et le cœur priment dans cette représentation. C'est par le sentiment que l'artiste apprend à mieux se connaître lui-même et à connaître le Beau, donc le Vrai, donc le Bien. Marie-Madeleine Fragonard dans sa présentation des Vies d'artistes, résume bien cette double école de l'art et de la vie qui débouche sur un univers de libération de l'être et d'enrichissement de la communication :

Bien sûr les artistes sont une aristocratie, mais ils ne font que déployer les forces que le malheur du monde ne permet pas d'épanouir chez tous : la possibilité d'être pleinement humains, c'est-à-dire divins, ouverts, heureux, en communion. En attendant le temps de la liberté, ils sont la liberté incarnée, en attendant la connaissance, ils sont la révélation, en attendant la communion des âmes, ils sont les auxiliaires d'une compassion sociale. Ils sont le Peuple encore inconscient d'être Peuple, uni' ${ }^{13}$.

Dans un dialogue à la fin du roman, le narrateur et Stella résument bien la portée de ce récit d'initiation individuelle et collective sur le plan de la métamorphose des êtres. En aidant Célio à se transformer, tous les autres personnages ont vécu eux aussi dans la générosité et la solidarité une transformation de leur être :

«Je joue ici la comédie comme Cécilia et son père la jouent; pour aider l'œuvre collective qui sert à l'éducation de Célio » avoue Stella. «Oui, il [Boccaferri] a mille fois raisons, répondis-je, et le cœur aussi s'ouvre à la poésie, à l'effusion, à l'amour, dans cette joyeuse et sympathique épreuve.» ( $C D$, p. 95I)

Le rêve d'unité et d'harmonie, se trouve en effet au bout de cette initiation au théâtre et à la vie. C'est lui qui emportait Consuelo sur les chemins de la connaissance et de l'initiation. Chaque représentation d'artiste généreux est marquée par ce rêve.

Le théâtre apparaît d'abord comme un miracle d'équilibre vivant, mouvant, entre la spontanéité, l'intuition, et la réflexion. Le théâtre permet surtout d'incarner ce rêve d'harmonie parce que tout se tient en lui : décor, costume, action, interprétation, mise en scène, musique. Au château des Désertes, chacun s'occupe librement avant la représentation selon son talent. Le héros narrateur fait de la peinture avec Stella, Célio fait l'éducation littéraire et musicale de son jeune frère et de sa jeune sœur Béatrice. Quant à la représentation elle-même, elle réunit plusieurs arts : la comédie et l'opéra.

J'avais cru, d'après le cœur, où faute d'instruments, des voix charmantes remplaçaient les combinaisons harmoniques de l'orchestre, qu'il s'agissait de l'opéra de

I3. M.-M. Fragonard, «Présentation», ouvr. cité, p. XIV-XV. 
Mozart rendu d'une certaine façon; mais le dialogue parlé de Célio et de Boccaferri me fit croire qu'on jouait la comédie de Molière en italien. Je la savais presque par cœur en français; je ne fus pas longtemps à m’apercevoir qu'on ne suivait pas cette version à la lettre. $(C D$, p. 913)

George Sand ne manque pas une occasion de souligner à quel point le théâtre est une œuvre collective. Tous les acteurs sont unis. Boccaferri invite ses disciples à «être frères dans l'art»( $C D$, p. 926).

La théorie de l'individualisme, qui règne au théâtre plus que partout ailleurs, et qui s'exerce en ignobles jalousies de métier pour souffler la claque à un camarade, est plus pernicieuse au talent sur les planches que sur toutes les autres scènes de la vie. $(C D$, p. 926$)$

\section{Il dit encore à ses élèves :}

Gardez-vous de la personnalité jalouse et pressée de se montrer! Gardez-vous-en comme de la peste! On ne s'éclaire qu'en s'écoutant les uns les autres. [...] Pour que chacun soit bon et vrai, il faut que tous le soient, et le succès qu'on ôte d'un rôle on l'ôte au sien propre [...] l'inspiration ne peut être que le résultat de la santé morale, elle ne descend que dans les âmes généreuses. (CD, p. 926)

Cette harmonie ne peut être trouvée que dans l'accord juste de toutes les voix. L'harmonie dans l'improvisation, mouvante et spontanée comme la vie, exige l'interdépendance de tous les participants, la solidarité infaillible du groupe, une interactivité vivante, une commune chaleur d'inspiration.

Le théâtre représente aussi une aspiration à réunir les classes sociales : artistes, aristocrates, peuple. «L'artiste devient lui-même un lien social où peuvent se retrouver à égalité et se confondre les deux classes jusqu'ici antagonistes de la noblesse et du peuple ${ }^{14} »$. Boccaferri, le génial chef de troupe du château des Désertes n'échappe pas, comme l'auteur, à cette double appartenance : populaire et aristocratique. L'aristocrate artiste est d'ailleurs artiste avant d'être aristocrate. Célio dit à Salvator, le narrateur, que Cécilia et son père «n'ont pas cessé d'être artistes» et «qu'ils le sont plus que jamais depuis qu'ils sont devenus seigneurs» ( $C D$, p. 940). Boccaferri lui-même en revenant sur son passé avoue : "J'oubliai même avec un certain plaisir que j'étais un patricien pour m’affermir dans la vie d'artiste, pour laquelle j'étais né» $(C D$, p. 935). Quant aux élèves de Boccaferri, les enfants très doués de la Floriani, elle-même issue du peuple, ils représentent cette nouvelle aristocratie qui peut accomplir ce rêve d'harmonie : celle de l'esprit et celle du don.

I4. N. Abdelaziz, Le personnage de l'artiste dans l'œuvre romanesque de G. Sand, thèse de doctorat, Clermont II, I996, p. I23. 
L'harmonie réside dans l'œuvre collective, elle réside aussi à la fin du roman, au bout de l'apprentissage, dans le cœur de ces artistes. Eux-mêmes, en parvenant à la connaissance, ont réussi à conquérir une forme d'unité, à concilier des tendances contradictoires de leur être, de leur génie. L'artiste sandien est toujours en quelque sorte androgyne, a souligné dans sa thèse Nathalie Abdelaziz ${ }^{15}$. Il allie comme la Floriani, comme la fille de Boccaferri, comme Célio, comme Salvator, les qualités dites féminines de la sensibilité et de l'intuition aux qualités dites viriles de décision et de création. Le personnage de l'artiste va découvrir au bout de son apprentissage le sens de la vie. Cette découverte n'est pas la conquête d'une harmonie figée, d'un centre fixe. S'il construit pour ses élèves une sorte d'abbaye de Thélème, le temps de leur apprentissage, Boccaferri sait que cette maison de formation idéale ne restera pas fermée comme un couvent.

Le personnage de l'artiste est un missionnaire qui après l'exploration de son art et de son moi, reprendra le chemin. Le bonheur auquel il parvient n'est ni individualiste, ni égoïste. En découvrant le sens de la vie, il souhaite que l'humanité retrouve un jour son unité. Sa marche annoncée, comme celle de Consuelo, est une marche symbolique. L'originalité de l'artiste sandien, qu'il soit comédien ou musicien, est d'être un personnage qui ne connaît pas une fin :

La fin sans fin est adaptée à la trajectoire d'apprentissage inséparable de la condition d'artiste qui franchit successivement les étapes de la maîtrise de l'outil et la technique, la formation progressive du talent et du moi, la découverte et l'acceptation du sens de la vie [...]. Même passé maître en son art, l'artiste n'en a jamais fini avec la création. Le propre de son art, c'est de se renouveler sans cesse ${ }^{16}$.

\section{C'était déjà la fin de La comtesse de Rudolstadt.}

C'est un trait majeur de l'imaginaire et de la pensée de cet écrivain qui se déploie autour de ces deux termes, otium et création. Chez Sand, ces deux termes ne sont pas antithétiques. Bien au contraire ils déclenchent une dynamique. Dans aucun de ces trois romans consacrés à des vies d'artistes, l'otium n'a coïncidé avec le vide. Pourtant dans ces trois romans l'otium représenté exprime bien d'abord une rupture avec la vie sociale et professionnelle de ces artistes. Cette rupture qui se manifeste d'une manière différente dans les trois exemples, puisqu'elle peut passer par le voyage ou la mobilité ou par le choix d'un refuge et de l'immobilité spatiale, est source de découvertes nouvelles sur l'art, sur soi, et va coïncider avec une expérience inattendue du plein. Il

I5. Ibid.

I6. Ibid., p. 4I2. 
$\mathrm{y}$ a quelque chose de très positif qui sort à chaque fois de ce recul, quelque chose de positif sur le plan de l'art et sur celui de l'épanouissement de l'être qui semble avoir mûri grâce à cette rupture et à l'absence de contrôle total qu'il y a eu dans cette rupture. L'alliance de l'otium et de la création dans l'œuvre de George Sand met en lumière deux traits majeurs de sa personnalité et de sa pensée : la liberté personnelle et la recherche du bonheur. 